\title{
Erratum \\ Representations of surface groups in the projective general linear group
}

[Int. J. Math., Vol. 22, No. 2 (2011) 223-279]

\author{
André Gama Oliveira \\ Departamento de Matemática \\ Universidade de Trás-os-Montes e Alto Douro \\ Quinta dos Prados, Apartado 1013 \\ 5000-911 Vila Real, Portugal \\ agoliv@uiad.pt
}

Published 26 February 2019

The purpose of this note is to point out a flawed argument in [1] which invalidates Theorem 9.1. This also affects the results in Sec. 2, namely, Theorems 11.1 (stated as Theorem 1.3 in the Introduction) and 11.2. However the main results of the paper - Theorems 1.1 and 1.2, concerning the number of connected components of the moduli space $\mathcal{M}_{\mathrm{PGL}(n, \mathbb{R})}$ of $\mathrm{PGL}(n, \mathbb{R})$-Higgs bundles over a compact Riemann surface $X$ - are completely unaffected by this flaw. Indeed, Secs. 9 and 11 only deal with the particular case of $\mathrm{SL}(3, \mathbb{R}) \cong \mathrm{PGL}(3, \mathbb{R})$, where we consider the topology (more precisely, the Poincaré polynomial) of $\mathcal{M}_{\mathrm{SL}(3, \mathbb{R})}$.

By the non-abelian Hodge correspondence, $\mathcal{M}_{\mathrm{SL}(3, \mathbb{R})}$ is homeomorphic to the $\mathrm{SL}(3, \mathbb{R})$-character variety $\mathcal{M}_{\mathrm{SL}(3, \mathbb{R})}$ of representations $\pi_{1} X \rightarrow \mathrm{SL}(3, \mathbb{R})$.

$\mathrm{SL}(3, \mathbb{R})$-Higgs bundles $(V, Q, \Phi)$ are topologically classified by the second Stiefel-Whitney class $w_{2} \in H^{2}\left(X, \mathbb{Z}_{2}\right) \cong \mathbb{Z}_{2}$ of the underlying rank 3 orthogonal bundle $(V, Q)$. Denote by $\mathcal{M}_{\mathrm{SL}(3, \mathbb{R})}\left(w_{2}\right)$ the subspace corresponding to $\mathrm{SL}(3, \mathbb{R})$ Higgs bundles with $w_{2}(V, Q)=w_{2}$. Then

$$
\mathcal{M}_{\mathrm{SL}(3, \mathbb{R})}=\mathcal{M}_{\mathrm{SL}(3, \mathbb{R})}(0) \sqcup \mathcal{M}_{\mathrm{SL}(3, \mathbb{R})}(1) .
$$

Moreover, $\mathcal{M}_{\mathrm{SL}(3, \mathbb{R})}(1)$ is connected, while

$$
\mathcal{M}_{\mathrm{SL}(3, \mathbb{R})}(0)=\mathcal{M}_{\mathrm{SL}(3, \mathbb{R})}^{\prime}(0) \sqcup \mathcal{M}_{\mathrm{SL}(3, \mathbb{R})}^{\prime \prime}(0)
$$


has two connected components, one of them - denoted here by $\mathcal{M}_{\mathrm{SL}(3, \mathbb{R})}^{\prime \prime}(0)$ being the celebrated Hitchin component. Call the other two components, namely, $\mathcal{M}_{\mathrm{SL}(3, \mathbb{R})}^{\prime}(0)$ and $\mathcal{M}_{\mathrm{SL}(3, \mathbb{R})}(1)$, the non-Hitchin components.

In Theorem 9.1, we claim that each of the two non-Hitchin components admits a deformation retraction onto the corresponding components of the moduli space of rank 3 orthogonal vector bundles (i.e. SO(3)-Higgs bundles), which are also distinguished by the second Stiefel-Whitney class. However, this claim is false. The reason is that, contrary to what is claimed on p. 273 , there are $\mathbb{C}^{*}$-fixed points in $\mathcal{M}_{\mathrm{SL}(3, \mathbb{R})}^{\prime}(0)$ and in $\mathcal{M}_{\mathrm{SL}(3, \mathbb{R})}(1)$ which are not local minima of the Hitchin functional.

Indeed, a fixed point $(V, Q, \Phi)$, with nonzero Higgs field, must be of the form

$$
V=F \oplus \mathcal{O} \oplus F^{-1},
$$

where $F$ is a line bundle, $Q$ is the obvious anti-diagonal quadratic form, and the Higgs field must be of one of the following forms:

$$
\Phi=\left(\begin{array}{ccc}
0 & 0 & 0 \\
\Phi_{1} & 0 & 0 \\
0 & \Phi_{1} & 0
\end{array}\right)
$$

with $\Phi_{1}: F \rightarrow K$ nonzero, or

$$
\Phi=\left(\begin{array}{ccc}
0 & 0 & 0 \\
0 & 0 & 0 \\
\Phi_{1} & 0 & 0
\end{array}\right),
$$

with $\Phi_{1}: F \rightarrow F^{-1} K$ nonzero. In the case of (11) and (2), we must have

$$
0 \leq \operatorname{deg}(F) \leq 2 g-2,
$$

while in the case of (1) and (3),

$$
0 \leq \operatorname{deg}(F) \leq g-1 \text {. }
$$

Among the above fixed points, only the ones of type (1) and (2) with $\operatorname{deg}(F)=$ $2 g-2$ (thus $F \cong K$ ) are local minima. These lie in the Hitchin component, which does not have any further fixed points (thus, it is contractible). Hence, the other fixed points lie in $\mathcal{M}_{\mathrm{SL}(3, \mathbb{R})}^{\prime}(0)$ or $\mathcal{M}_{\mathrm{SL}(3, \mathbb{R})}(1)$. Indeed, it is easy to check that these fixed points have $w_{2}(V, Q)=\operatorname{deg}(F) \bmod 2$, hence they exist both in $\mathcal{M}_{\mathrm{SL}(3, \mathbb{R})}^{\prime}(0)$ and $\mathcal{M}_{\mathrm{SL}(3, \mathbb{R})}(1)$, depending on the degree of $F$.

This implies that the moduli space of rank 3 special orthogonal bundles (which has two components distinguished by $w_{2}$ ), is not a deformation retraction of the space $\mathcal{M}_{\mathrm{SL}(3, \mathbb{R})}^{\prime}(0) \sqcup \mathcal{M}_{\mathrm{SL}(3, \mathbb{R})}(1)$ given by the non-Hitchin components. Hence, Theorems 9.1, 11.1 (and 1.3) and 11.2 do not hold.

\section{Reference}

[1] A. Oliveira, Representations of surface groups in the projective general linear group, Int. J. Math. 22(2) (2011) 223-279. 